\title{
To Assess the Efficacy of the Measures Taken to Reduce the Primary Caesarean Rates and Its Impact on Overall Caesarean Rate in a Tertiary Care Hospital
}

\author{
S Prajwal ${ }^{1}$, Rao PS ${ }^{1}$, Mitra Sujoy ${ }^{1}$, Mukhopadhyay Indrani ${ }^{1}$, \\ Sharma Arunav ${ }^{1}$, Jain Charu ${ }^{1}$ \\ ${ }^{I}$ Department ofObstetrics andGynaecology, Command Hospital, Bangalore
}

\begin{abstract}
:
Introduction: Caesarean delivery defines the birth of a fetus via laparotomy and then hysterotomy. Caesarean section rates have continued to increase worldwide in recent years. The reasons for increase in the caesarean delivery rates may be due to increase in number of elderly primi gravidae, caesarean delivery on maternal request, fear of litigation in obstetric practice and the reluctance on the part of obstetricians and the patients to accept even minute errors and many more. Rise in caesarean section rate should be assessed for improving quality of care for the pregnant woman. We evaluated the rate of caesarean births according to Robson criteria in our birth centre and aimed to determine the cause of this rising trend and assess the efficacy of various measures taken to reduce the rate of caesarean section.

Methods:The study was conducted in a Command Hospital of the Armed Forces which is a tertiary care centre. Data of the patients who delivered between January 2014 to December 2016 were collected from labour room register and those women who delivered by caesarean section were classified according to Robson's classification. As strict measures were instituted to reduce the rates as per the ACOG Obstetric care consensus at the beginning of the year 2016 we undertook to assess the efficacy of those measures in comparison to the previous two years. The rates were calculated for all the three years according to the various groups as per Robson's classification.

Results:Total number of deliveries in 2014, 2015 and 2016 were 984, 973 and 980 respectively, out of which the annual caesarean rate in 2014 was $30.38 \%, 2015$ was $30.6 \%$ and 2016 was $28.57 \%$ and the average rate in last three years was 29.86\%. The caesarean rate was higher than stipulated WHO 15\%. The percentage of patients falling into Robson-2 was $20.46 \%, 16.11 \%$ and 11.07\% in the year 2014, 2015 and 2016 respectively which showed a significant reduction. The percentage of patients falling into Robson-4 showed significant reduction in the rate of caesarean section in multiparous women who had previous vaginal delivery $(4.01 \%, 3.36 \%$ and $0.36 \%$ respectively). The major percentage of caesarean section rates was in Robson-5 group, $57.68 \%, 64.43 \%$ and $72.50 \%$ for the three years respectively. Regarding the Robson-8 group we determined that there was an increase in vaginal delivery for twin pregnancy. Robson-10, showed decreasing trend with complete reduction of preterm delivery $(4.68 \%, 1.34 \%$ and $0.0 \%$ respectively) which was statistically significant. Our study has found that there was a fall in the caesarean section rate in the year 2016 as compared to the preceding 2 years.

Conclusion: Various measures were taken to reduce the primary caesareans as per the ACOG Obstetric care consensus. The caesarean rate in 2014 was $30.38 \%, 2015$ was $30.62 \%$ and 2016 was $28.57 \%$ and the reduction in primary caesarean rate contributed significantly to the decrease in overall caesarean rate by $2.05 \%$ in 2016.
\end{abstract}

Keywords: ACOG Obstetric Consensus Caesarean section rates Robson's classification

\section{Introduction}

Caesarean section rate has been increasing worldwide over the years and the rate of increase is highest in low income countries [1]. Caesarean section is the most significant delivery event. Therefore, it will definitely be at the centre of discussion and the worldwide rise in caesarean rates is a major public health concern and has raised a professional debate due to potential maternal and perinatal risks, cost issues and inequity in access.[2,3] WHO and the US Healthy People 2000 initiative, suggested $10-15 \%$ as the optimal caesarean section rate. [4] It is however difficult to determine optimal rates for institutions, especially referral centres.

It has been suggested that caesarean section rates should no longer be taken as very high or very low but rather whether they are appropriate or not, after taking into consideration all relevant information. [5] To capture all relevant information about the various reasons for the caesarean delivery the Robson classification with various modifications has been put forward and been used in many centers worldwide. The Robson criteria is a ten group classification system (RTGCS) using 10 mutually exclusive and totally inclusive categories for caesarean section i.e. any women can only be classified into only one group. Robson's classification depends on women's gestation age, onset of labour, fetal presentation and number of fetuses without the knowledge of the indication for induction of labour. Categories are totally inclusive and mutually exclusive. It can be easily classified and it can provide the critical assessment of care at delivery. Information obtained by Robson's classification helps delivery units for understanding and help in better care of women.

\begin{tabular}{|l|l|}
\multicolumn{2}{l}{ Robsons classification (Table 1) } \\
\hline Number & Group \\
\hline 01 & Nulliparous, single cephalic, $>37$ weeks in spontaneous labor \\
\hline 02 & Nulliparous, single cephalic, $>37$ weeks, induced or CS before labor \\
\hline 03 & Multiparous (excluding previous CS), single cephalic, $>37$ weeks in spontaneous labor \\
\hline 04 & $\begin{array}{l}\text { Multiparous (excluding previous CS), single cephalic, }>37 \text { weeks, induced or CS before } \\
\text { labor }\end{array}$ \\
\hline
\end{tabular}


To Assess The Efficacy Of The Measures Taken To Reduce The Primary Caesarean...

\begin{tabular}{|l|l|}
\hline 05 & Previous CS, single cephalic, $>37$ weeks \\
\hline 06 & All nulliparous breeches \\
\hline 07 & All multiparous breeches (including previous CS) \\
\hline 08 & All multiple pregnancies (including previous CS) \\
\hline 09 & All abnormal lies (including previous CS) \\
\hline 10 & All single cephalic, $<36$ weeks (including previous CS) \\
\hline
\end{tabular}

We classified the caesarean sections according to Robson's criteria to interpret and to know the various rates and trends of caesarean section and decided to take action as per the ACOG Obstetric care consensus to reduce the caesarean rates.

\section{Materials And Methods}

Data was collected from the labour room register. All women delivered during the period from January 2014 to December 2016 were included. Relevant information as parity, mode of previous delivery, gestational age, and onset of labour, spontaneous or induced, indication for caesarean and operative notes were obtained and evaluated from the previous case sheets of the patients. In the year 2016 strict measures as per the ACOG Obstetric care consensus were instituted and the patients were allotted groups according to the Robson classification. We strictly followed the above guidelines in the following manner:-

2.1. Preinduction cervical ripening was started from $40 \mathrm{wk} 3$ days period of gestation and avoided unnecessary caesarean deliveries due to unfavorable cervix.

2.2. Cervical ripening methods were used when labor is induced in women with an unfavorable cervix.

2.3.If the maternal and fetal status allowed caesarean deliveries for failed induction of labor in the latent phase were avoided by allowing longer durations of the latent phase (up to 24 hours or longer) and oxytocin was administered for at least 12-18 hours after membrane rupture before deeming the induction a failure.

2.4. In our hospital we employed proper labour management to reduce the CS rate. Achieving a good uterine contraction, proper usage of oxytocin drip, use of partogram and proper dystocia treatment, fetal monitoring in the labour unit were all instituted.

2.5. We ensured that slow but progressive labor in the first stage of labor should not be an indication for caesarean delivery and cervical dilation of $6 \mathrm{~cm}$ should be considered the threshold for the active phase of most women in labor. Thus, before $6 \mathrm{~cm}$ of dilation is achieved, standards of active phase progress were not applied.

2.6. Caesarean delivery for active phase arrest in the first stage of labor was reserved for women at or beyond $6 \mathrm{~cm}$ of dilation with ruptured membranes who fail to progress despite 4 hours of adequate uterine activity, or at least 6 hours of oxytocin administration with inadequate uterine activity and no cervical change.

2.7. A specific absolute maximum length of time spent in the second stage of labour beyond which all women should undergo operative delivery has not been specified. Before diagnosing arrest of labor in the second stage, if the maternal and fetal conditions permit, we allowed for the following: At least 2 hours of pushing in multiparous women and at least 3 hours of pushing in nulliparous women.

2.8. Operative vaginal delivery in the second stage of labor by experienced and well trained physicians was used as an option as it is safe, acceptable alternative to caesarean delivery.

2.9. Manual rotation of the fetal occiput in the setting of fetal malposition in the second stage of labor is a reasonable intervention which was always tried before moving to operative vaginal delivery or caesarean delivery.

We calculated the rates as per each group and analyzed if there was any effect of the measures instituted over a period of one year on the rate of caesarean section as well as on the rate of primary caesarean section rates.

Observations and Results

Total number of women delivered during the period was 2937 out of which 877 were Caesarean sections. These women were categorized according to Robson's classification Groups and analyzed.

Distribution of caesarean as per the year (Table 2)

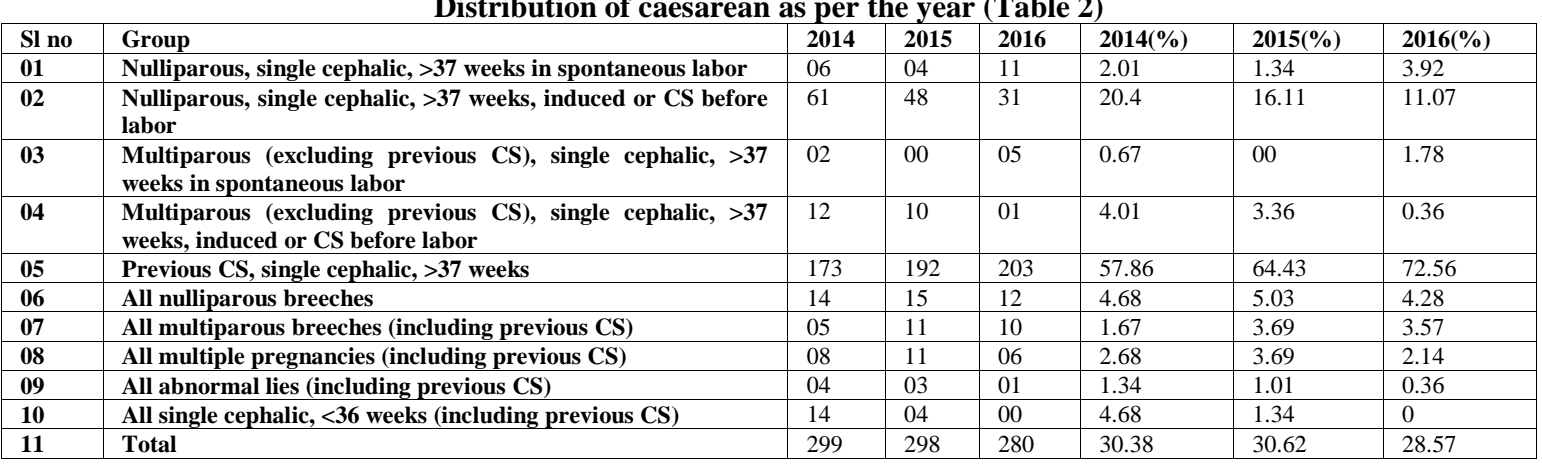

Table 2 shows the number of cases and percentage of caesarean in each group. In group 1 the number of caesarean cases were $06(2.01 \%), 04(1.34 \%)$ and $11(3.92 \%)$ in the year 2014, 2015 and 2016 respectively.

In group 2 the number of caesarean cases were 61 (20.4\%), 48 (16.11\%) and 31(11.07\%) in the year 2014, 2015 and 2016 respectively which is showing the decreasing trend, which is statistically significant ( $\mathrm{p}$ value $=0.023$ ).

In group 3 the number of caesarean cases were $02(0.67 \%), 00(00 \%)$ and 05(1.78\%) in the year 2014, 2015 and 2016 respectively. 
In group 4 the number of caesarean cases were $12(4.01 \%), 10(3.36 \%)$ and 04(0.36\%) in the year 2014, 2015 and 2016 respectively with decreasing trend and is statistically significant ( $\mathrm{p}$ value $=0.015)$.

In group 5 the number of caesarean cases were $176(57.86 \%), 192(64.43 \%)$ and 203(72.5\%) in the year 2014, 2015 and 2016 respectively, which is showing increasing trend but not statistically significant.

In group 6 the number of caesarean cases were 14 (4.68\%), 15 (5.03\%) and 12(4.28\%) in the year 2014, 2015 and 2016 respectively.

In group 7 the number of caesarean cases were 05 (1.67\%), 11 (3.69\%) and 10 (3.57\%) in the year 2014, 2015 and 2016 respectively.

In group 8 the number of caesarean cases were 8 (2.68\%), 11 (3.69\%) and 06(2.14\%) in the year 2014, 2015 and 2016 respectively.

In group 9 the number of caesarean cases were $04(1.34 \%), 03(1.01 \%)$ and 1(0.36\%) in the year 2014, 2015 and 2016 respectively.

In group 10 the number of caesarean cases were 14 (4.68 \%), $04(1.34 \%)$ and $00(00 \%)$ in the year 2014, 2015 and 2016 respectively, significant statistically ( $\mathrm{p}$ value $=0.00$ ).

The overall caesarean rate was $29.86 \%$ over the last three years with $30.38 \%$ in 2014, 30.62\% in 2015 and $28.57 \%$ in 2016 .

Caesarean rates in Year 2014, 2015 and 2016 (Figure 1)

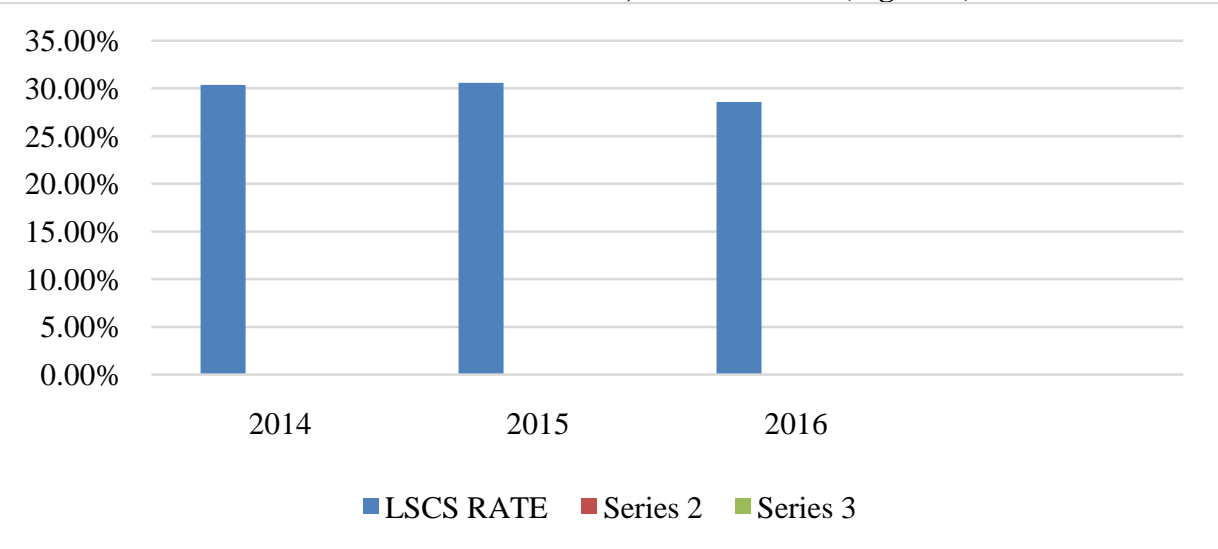

Fig 1 shows the rate of caesarean in the year 2014, 2015 and 2016.The caesarean rate in 2014 was 30.38\%,2015 was $30.62 \%$ and 2016 was $28.57 \%$ and the overall rate in last three years is $29.86 \%$.

Comparison of caesarean rates as per the groups in Year 2014, 2015 and 2016 (Figure 2)

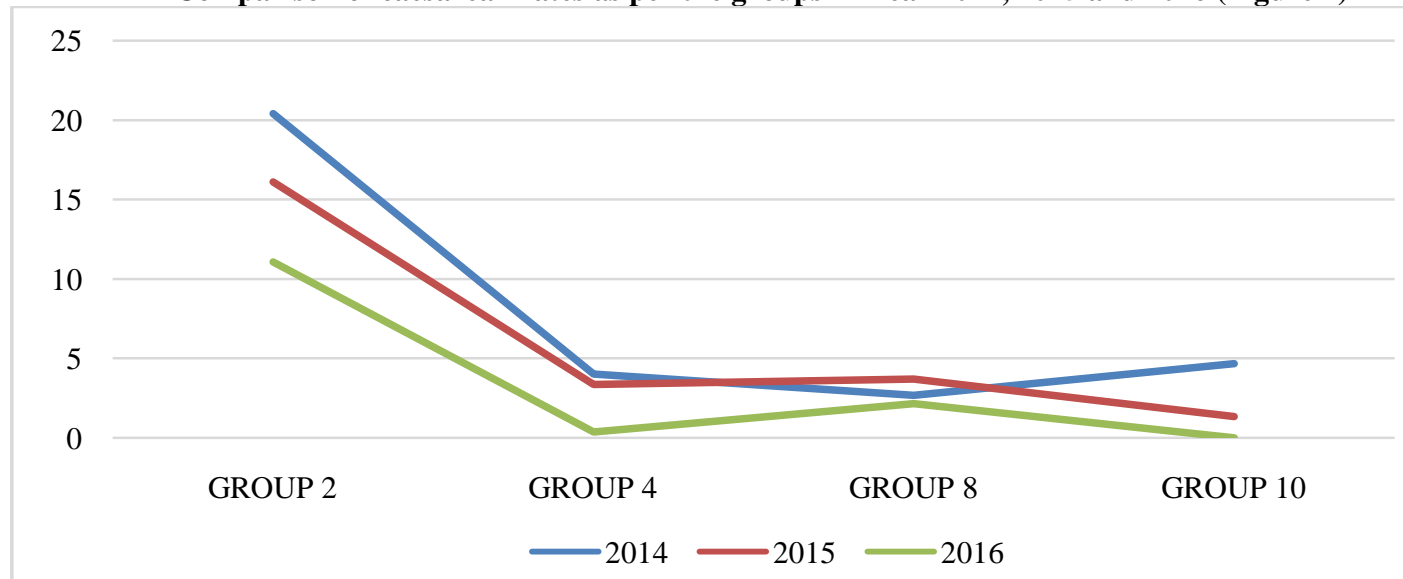

Fig 2 shows the, rates of caesarean in group 2, 4, 8 and 10, which shows the decreasing trend in the year 2016 in all the groups

\section{Discussion}

Overall 3 year average caesarean section rate observed at our centre was $29.86 \%$. It was higher than WHO criteria of $15 \%$ caesarean section (CS) rate, but lesser than Tasmania (33\%), Queens land and slightly greater than Australia (28\%)[6,7], 27\% in United States of America,[8]Asian countries 27.3\%[9]. There was decreasing trend in caesarean section rate from 2014 to 2016 from $30.38 \%$ to $28.58 \%$ as efforts and measures as per ACOG Obstetric care consensus were made to reduce the caesarean section rates especially the primary caesarean rates.

Contribution of Group 1 to the total caesarean rate in all the 3 years was very little. The contribution of Group 2 to the CS rate was $20.4 \%$ in 2014, $16.11 \%$ in 2015 and $11.07 \%$ in 2016. There is a significant reduction in caesarean rate over 3 years which is significant statistically. As per the ACOG Obstetric care consensus [10] based on maternal and fetal medical indications. Inductions at 41 0/7 weeks of gestation and beyond should be performed to reduce the risk of caesarean delivery 
and the risk of perinatal morbidity and mortality. Cervical ripening methods should be used when labor is induced in women with an unfavorable cervix. If the maternal and fetal status allow, caesarean deliveries for failed induction of labor in the latent phase can be avoided by allowing longer durations of the latent phase (up to 24 hours or longer) and requiring that oxytocin be administered for at least 12-18 hours after membrane rupture before deeming the induction a failure. In our hospital we strictly follow the above guidelines and preinduction cervical ripening is started from 40wk 3day period of gestation and avoid unnecessary caesarean deliveries.

Proper labour management is necessary to reduce the caesarean rate in this group. Achieving a good uterine contraction, proper usage of oxytocin drip, use of partogram and proper dystocia treatment, fetal monitoring in the labour unit will reduce the caesarean section rate in this group. As per the ACOG Obstetric care consensus [10] - a prolonged latent phase (e.g. 20 hrs. in prim gravida and 14 hours in multiparous women) should not be an indication for caesarean delivery, slow but progressive labor in the first stage of labor should not be an indication for caesarean delivery and cervical dilation of $6 \mathrm{~cm}$ should be considered the threshold for the active phase of most women in labor. Thus, before $6 \mathrm{~cm}$ of dilation is achieved, standards of active phase progress should not be applied. Caesarean delivery for active phase arrest in the first stage of labor should be reserved for women at or beyond $6 \mathrm{~cm}$ of dilation with ruptured membranes who fail to progress despite 4 hours of adequate uterine activity, or at least 6 hours of oxytocin administration with inadequate uterine activity and no cervical change. These measures are to be taken to reduce the incidence of primary caesarean rates.

A specific absolute maximum length of time spent in the second stage of labour beyond which all women should undergo operative delivery has not been specified. Before diagnosing arrest of labor in the second stage, if the maternal and fetal conditions permit, allow for the following: At least 2 hours of pushing in multiparous women and at least 3 hours of pushing in nulliparous women. Longer durations may be appropriate on an individualized basis (e.g., with the use of epidural analgesia or with fetal malposition) as long as progress is being documented. Operative vaginal delivery in the second stage of labor by experienced and well trained physicians should be considered a safe, acceptable alternative to caesarean delivery. Training in, and ongoing maintenance of, practical skills related to operative vaginal delivery should be encouraged. Manual rotation of the fetal occiput in the setting of fetal malposition in the second stage of labor is a reasonable intervention to consider before moving to operative vaginal delivery or caesarean delivery. In order to safely prevent caesarean deliveries in the setting of malposition, it is important to assess the fetal position in the second stage of labor, particularly in the setting of abnormal fetal descent. All the above recommendations were strictly followed.

The contribution of Group 3 to the caesarean section rate should be less than 3\%. [11] It should be similar in every labour unit. In our study it is $0.67 \%$ in $2014,00 \%$ in 2015 and $1.78 \%$ in 2016 which is within in the acceptable range and in fact better than the acceptable percentage.

The contribution of Group 4 to the caesarean section rate should be 5-8\%.[11] Our centre had the following rates in the last 3 years that is $4.01 \%$ in $2014,3.36 \%$ in 2015 and $0.36 \%$ in 2016 . There is a significant reduction in caesarean rate in the multiparous women who had previous vaginal delivery and is statistically significant.

The contribution of Group 5 to the caesarean rate was $57.86 \%$ in $2014,64.43 \%$ in 2015 and $72.56 \%$. It is the largest group in all the three years as a percentage contribution to the overall annual caesarean rates. The rate in the year 2016 looks as if it has obviously increased because the contribution from other groups has significantly fallen but this increase was not statistically significant either. It is obvious that the main aim of instituting measures to reduce caesarean section rates is to firstly reduce the primary caesarean rates. The caesarean section rates of Group 5 can be reduced by proper selection of patients for Trial of Labour after Caesarean (TOLAC) and Vaginal Birth AfterCaesarean (VBAC).

The contribution of Group 6 to the caesarean rate was $4.68 \%$ in 2014, 5.03\% in 2015 and $4.28 \%$ in 2016, As per ACOG obstetric care consensus fetal presentation should be assessed and documented beginning at $360 / 7$ weeks of gestation to allow for external cephalic version to be offered. In our institution we offer external cephalic version for all the patients who fulfil the pre-requisites of extern al cephalic version.

The contribution of Group 7 to the caesarean rate was $1 \%$ in $2014,3.69 \%$ in 2015 and $3.57 \%$ in 2016 which is less than Group 6, as the success rate of external cephalic version is more in multigravida.

The contribution of Group 8 to the caesarean rate was $1.34 \%$ in $2014,1.01 \%$ in 2015 and $0.36 \%$ in 2016 . It is a heterogeneous group, contributing less to the overall CS rate, has a significantly high perinatal mortality rate. Route of delivery depends on the chorionicity, presenting part of the first twin. The reason for the reduction in caesarean rate in this group is mainly because of strict measures employed to increase the rate of vaginal delivery for twins.

The contribution of Group 9 to the caesarean rate was $1.34 \%$ in $2014,1,01 \%$ in 2015 and $0.36 \%$ in 2016 which was very minimal.

The contribution of Group 10 to the caesarean rate was also reduced over the 3 years although $10 \%$ is normal. Caesarean rate was $4.68 \%$ in 2014, 1.34\% in 2015 and $00 \%$ in 2016 suggest that there is a complete elimination of preterm caesarean which significantly decreases the future caesarean delivery.

In our centre, although caesarean delivery rate is higher than WHO accepted rate, there has been a significant attempt made to reduce primary caesarean rates and overall caesarean rate reduced by $2.01 \%$ in 2016 , due to appropriate measures which were instituted.

\section{Conclusion}

The caesarean rate in 2014 was $30.38 \%$, in 2015 was $30.62 \%$ and in 2016 was $28.57 \%$ and the overall rate in the last three years was $29.86 \%$ and there was a reduction in the overall rate by $2.05 \%$ between 2014 and 2016 . Various measures were taken to reduce the primary caesareans in the form of proper monitoring of first stage labour, identification and correction of delay in labour and also application of instruments in second stage of delivery if required to reduce the rate of primary caesarean section. Pre induction cervical ripening was done before the induction of labour to prevent the failure of labour. External cephalic version was offered to all eligible patients. ACOG Consensus criteria for labour were applied and strictly followed. The number of caesareans in multiparous women as well as those with multiple pregnancy were 
reduced. The number of preterm caesareans were completely eliminated. All the above measures has resulted in decrease of primary caesarean rate which we believe is the way forward in bringing down the menace of increased caesarean rates all over the world.

\section{References}

[1]. Tapia V, Betran AP, Gonzales GF (2016) Caesarean Section in Peru: Analysis of Trends using the Robson Classification System. PLoS ONE 11:0148138

[2]. Gonzales GF, Tapis VL, Fort AC, Betran AP (2013), Pregnancy outcomes associated with Caesarean delivery in Peruvian public health facilities. Int J Women health 5:637-645

[3]. Torloni MR, Betran AP, Windmore M, Allen T, et L (2011). Classification for caesarean section: A system review PLos 6:e14566

[4]. WHO (1985), Appropriate technology for birth, Lancet 24:4360-4370.

[5]. Robson MS (2001), Can we reduce the caesarean section rate? Best Pract Res Clin Obstet Gynaecol 15; 179-194.

[6]. Stavrou EP, Ford JB, Shand AW, Morris JM, Roberts CL. Epidemiology and trends for Caesarean section births in New South Wales, Australia: a population-based study. BMC Pregnancy Childbirth 2011;11:8 10.1186/1471-2393-11-8 [PMC free article] [PubMed] [Cross Ref]

[7]. Laws PJ, Sullivan EA. Australia's mothers and babies 2007. 2009. Sydney.

[8]. Cyr RM.Myth of ideal caesarean section rate: commentary and historic prospective. Am J Obstet Gynaecol.2006; 194:932-6.

[9]. Lumbiganon P, Laopaiboon M, Gulmezoglu AM, Souza JP, Taneepanichskul S, Ruyan P, Attygalle DE, Shrestha N, Mori R, Nguyen DH, Method of delivery and pregnancy outcomes in Asia: the WHO global survey on maternal and perinatal health 200708. Lancet. pp. 490-499. [PubMed].

[10]. Safe prevention of the primary caesarean delivery. Obstetric Care Consensus No.1 American College of Obstetricians and Gynecologists, Obstet Gynecol 2014:123-698-711.

[11]. Grace Neville, Micheal Robson, Caesarean Section Rates: Much Ado about Nothing or a Marker of Quality Care?,in John Studd, Seang Lin Tan. Frank A.Chevenak, Current progress in Obstetrics\&Gynaecology Vol-2 (Mumbai: Tree Life Media - Kothari Medical Subscription Services Pvt. Ltd.2014) 151-162. 